\title{
STABILIZATION OF THE KAWAHARA EQUATION WITH LOCALIZED DAMPING
}

\author{
Carlos F. Vasconcellos ${ }^{1}$ and Patricia N. Da Silva ${ }^{1}$
}

\begin{abstract}
We study the stabilization of global solutions of the Kawahara (K) equation in a bounded interval, under the effect of a localized damping mechanism. The Kawahara equation is a model for small amplitude long waves. Using multiplier techniques and compactness arguments we prove the exponential decay of the solutions of the $(\mathrm{K})$ model. The proof requires of a unique continuation theorem and the smoothing effect of the $(\mathrm{K})$ equation on the real line, which are proved in this work.
\end{abstract}

Mathematics Subject Classification. 35Q35, 35B40, 35Q53.

Received September 8, 2008. Revised January 27, 2009.

Published online October 30, 2009.

\section{INTRODUCTION}

We consider the Kawahara $(\mathrm{K})$ system in a bounded interval $(0, L)$ under the presence of a localized damping

$$
\mid \begin{aligned}
& u_{t}+u_{x x x}+\eta u_{x x x x x}+u u_{x}+a(x) u=0 \quad \text { in }(0, L) \times(0,+\infty) \\
& u(0, t)=u(L, t)=0 \quad \text { for all } t \geq 0 \\
& u_{x}(0, t)=u_{x}(L, t)=0 \quad \text { for all } t \geq 0 \\
& u_{x x}(L, t)=0 \quad \text { for all } t \geq 0 \\
& u(x, 0)=u_{0} \quad \text { in }(0, L) .
\end{aligned}
$$

Here $a=a(x)$ is a non-negative function belonging to $L^{\infty}(0, L)$ and moreover, in most of the paper we will assume that $a(x) \geq a_{0}>0$ a.e. in an open, non-empty subset $\omega$ of $(0, L)$, where the damping is acting effectively. The constant $\eta$ is a negative real number. The above system, in absence of damping (i.e. $a \equiv 0$ ), describes the one-dimensional evolution of small amplitude long waves in several problems arising in fluid dynamics.

In this model the conservative dispersive effect is represented by the term $\left(u_{x x x}+\eta u_{x x x x x}\right)$. The Kawahara equation is given by

$$
u_{t}+u u_{x}+\alpha u_{x x x}+\beta u_{x x x x x}=0
$$

where $\alpha$ and $\beta$ are constants representing the effect of dispersion. This equation is a model for plasma wave, capillarity-gravity water waves and other dispersive phenomena when the cubic KdV-type is weak. Kawahara [10] pointed out that it happens when the coefficient of the third order derivative in the KdV equation

\footnotetext{
Keywords and phrases. Kawahara equation, stabilization, energy decay, localized damping.

1 Instituto de Matemática e Estatística - UERJ, 524 R. São Francisco Xavier, Sala 6016, Bloco D - CEP 20550-013,

Rio de Janeiro, Brazil. cfredvasc@ime.uerj.br; nunes@ime.uerj.br
} 
becomes very small or even zero. It is necessary to take account of the higher order effect of dispersion in order to balance the nonlinear effect. Kakutani and Ono [9] showed that for a critical value of angle between the magneto-acoustic wave in a cold collision-free plasma and the external magnetic field, the third order derivative term in the $\mathrm{KdV}$ equation vanishes and may be replaced by the fifth order derivative term. Following this idea, Kawahara [10] studied a generalized nonlinear dispersive equation which has a form of the KdV equation with an additional fifth order derivative term. This equation has also been obtained by Hasimoto [8] for the shallow wave near critical values of surface tension. More precisely, in this work Hasimoto found these critical values when the Bond number is near one third.

While analyzing the evolution of solutions of the water wave-problem, Schneider and Wayne [23] also showed that the coefficient of the third order dispersive term in nondimensionalized statements of the KdV equation vanishes when the Bond number is equal to one third. The Bond number is proportional to the strength of the surface tension and in the $\mathrm{KdV}$ equation it is related to the leading order dispersive effects in the water-waves problem. With its disappearance, the resulting equation is just Burger's equation whose solutions typically form shocks in finite time. Thus, if we wish to model interesting behavior in the water-wave problem it is necessary to include higher order terms. That is, it is necessary to consider the Kawahara equation. In any case, the inclusion of the fifth order derivative term takes in account the comparative magnitude of the coefficients of the third and fifth power terms in the linearized dispersion relation.

Berloff and Howard [2] presented the Kawahara equation as the purely dispersive form of the following nonlinear partial differential equation

$$
u_{t}+u^{r} u_{x}+a u_{x x}+b u_{x x x}+c u_{x x x x}+d u_{x x x x x}=0 .
$$

The above equation describes the evolution of long waves in various problems in fluid dynamics. The Kawahara equation corresponds to the choice $a=c=0$ and $r=1$ and describes water waves with surface tension. Bridges and Derks [5] present the Kawahara equation - or fifth-order KdV-type equation - as a particular case of the general form

$$
u_{t}+\alpha u_{x x x}+\beta u_{x x x x x}=\frac{\partial}{\partial x} f\left(u, u_{x}, u_{x x}\right)
$$

where $u(x, t)$ is a scalar real valued function, $\alpha$ and $\beta \neq 0$ are real parameters and $f\left(u, u_{x}, u_{x x}\right)$ is some smooth function. The form (1.1) occurs most often in applications and corresponds to the choice of $f$ in (1.2) with form $f\left(u, u_{x}, u_{x x}\right)=-u^{2}$.

As noted by Kawahara [10], we may assume without loss of generality that $\beta<0$ in (1.1). In fact, if we introduce the following simple transformations

$$
u \rightarrow-u, \quad x \rightarrow-x \text { and } t \rightarrow t
$$

we can obtain an equation of the form of equation (1.1) in which $\alpha$ and $\beta$ are replaced, respectively, by $-\alpha$ and $-\beta$.

For further considerations see also, for example, Topper and Kawahara [24].

Nonlinear dispersive problems have been object of intensive research (see, for instance, the classical paper of Benjamin et al. [1], Biagioni and Linares [3], Bona and Chen [4], Menzala et al. [15], Rosier [19], and references therein). Recently global stabilization of the generalized KdV system has been obtained by Rosier and Zhang [20]. Linares and Pazoto [12] studied the stabilization of the generalized KdV system with critical exponents. Unique continuation and decay for the Korteweg-De Vries equation with localized damping have been studied by Pazoto [16].

For controllability problems involving dispersive systems, we can consider the work of Russel and Zhang [21] about KdV system and the paper by Linares and Ortega [11], where the Benjamin-Ono equation has been analyzed.

In Doronin and Larkin [7], using semi-discretization method, the existence and uniqueness of a regular solution for the Kawahara system were proved when the initial data belongs to $H^{5}(0,1)$. Its relation to a $\mathrm{KdV}$-solution was also established. Moreover the exponential decay of the energy for small initial data it was shown. 
In this paper, following from close the works by Menzala et al. [15] and Rosier [19] on the KdV equation, we study the problem of the decay of the energy for the $(\mathrm{K})$ system as $t \rightarrow+\infty$, in the presence of the localized damping term $a(x) u$.

We denote by $\|f\|$ the $L^{2}(0, L)$-norm of the function $f$.

The total energy associated with the $(\mathrm{K})$ system is defined by

$$
E(t)=\frac{1}{2} \int_{0}^{L}|u(x, t)|^{2} \mathrm{~d} x=\frac{1}{2}\|u(t)\|^{2} .
$$

Using the above boundary conditions we prove that

$$
\frac{\mathrm{d} E}{\mathrm{~d} t}=\frac{\eta}{2}\left|u_{x x}(0, t)\right|^{2}-\int_{0}^{L} a(x)|u(x, t)|^{2} \mathrm{~d} x \leq 0, \forall t>0 .
$$

So, $E(t)$ is a nonincreasing function of time. This paper is devoted to analyze the following questions: Does the energy $E(t) \rightarrow 0$ as $t \rightarrow+\infty$ ? Is it possible to find a rate of decay of the energy?

We observe that, according to the above energy dissipation law, even when $a \equiv 0, E(t)$ is a nonincreasing function.

The linearized problem, in which the term $u u_{x}$ is missing and the damping $a \equiv 0$, was analyzed by Vasconcellos and da Silva [25,26]. It has been proved that the linear semigroup decays exponentially for all values of the length of the space-interval $L$ and of $\eta<0$.

In [25] this result was proved by excluding a countable set of critical lengths $L$ but later on in [26] it was shown that the same holds for all $L$, unlike the case of the $\mathrm{KdV}$ in which there is effectively a countable set of lengths for which the energy of the corresponding linear system does not decay (see $[15,19])$.

In this work we study the nonlinear $(\mathrm{K})$ system.

In Section 2 we consider the initial data belonging to $L^{2}(0, L)$ then, using semigroup theory and multipliers techniques we prove the existence and uniqueness of global solutions for the (K) system in $C\left([0,+\infty) ; L^{2}(0, L)\right) \cap$ $L_{\text {loc }}^{2}\left(0,+\infty ; H_{0}^{2}(0, L)\right)$.

The Section 3 is devoted to study the stabilization of the $(\mathrm{K})$ system. Firstly, as a consequence of exponential decay of the energy associated to the linear equation, we prove the exponential decay of the small amplitude solutions of the nonlinear problem for all lengths $L$, even in absence of the damping $a$.

Then, we address to the problem of decay for large solutions. In this case we need to consider the extra damping $a=a(x)$ with the following assumptions: $a$ it is a non-negative function belonging to $L^{\infty}(0, L)$ and moreover that $a(x) \geq a_{0}>0$ a.e. in an open, non-empty subset $\omega$ of $(0, L)$, where the damping is acting effectively.

We argue using multipliers techniques and compactness arguments to handle the extra terms that the nonlinearity produces. We prove that all solutions of $(\mathrm{K})$ decay exponentially as $t \rightarrow+\infty$ with a decay rate, which is uniform in bounded sets of $L^{2}(0, L)$.

In our proof, we need a consequence of a theorem due to Saut and Scheurer [22] which show that the unique continuation principle for Kawahara system holds and moreover it is important to consider the smoothing effect of the $(\mathrm{K})$ equation on the real line (see Sect. 4).

In Section 4, inspired by an argument developed in Pazoto [16] for KdV problem, which was used before for linear, time invariant wave and plate equations respectively by Rauch and Taylor [18] and Zuazua [27], we prove a gain of regularity for solutions of the problem $(\mathrm{K})$ with $u_{x x}(0, t)=0$ that vanish on a subset $\omega$ contained in $(0, L)$.

Finally, in Section 5, we do some remarks about the Kawahara system.

Before we present our results, it is convenient to comment on the absence of the spatial derivative term $u_{x}$ in the Kawahara problem $(\mathrm{K})$. 
When we working on the whole real line, the linear transport term $u_{x}$ can be scaled out by a change of variables.

Although the term $u_{x}$ makes a physical sense in bounded domains, however in the proof of the results presented in this work, this term plays no essential role, even in presence of a damping. For further considerations, see Section 5 (Final remarks).

\section{EXISTENCE AND UNIQUENESS}

In this section we study the global existence and uniqueness of the solutions of the following problem

$$
\mid \begin{aligned}
& u_{t}+u_{x x x}+\eta u_{x x x x x}+u u_{x}+a(x) u=0 \quad \text { in }(0, L) \times(0,+\infty) \\
& u(0, t)=u(L, t)=0 \quad \text { for all } t \geq 0 \\
& u_{x}(0, t)=u_{x}(L, t)=0 \quad \text { for all } t \geq 0 \\
& u_{x x}(L, t)=0 \quad \text { for all } t \geq 0 \\
& u(x, 0)=u_{0} \quad \text { in }(0, L),
\end{aligned}
$$

where $a=a(x)$ is a non-negative function belonging to $L^{\infty}(0, L)$, satisfying $a(x) \geq a_{0}>0$ a.e. in an open, non-empty subset $\omega$ of $(0, L)$ and $\eta$ is a negative real number.

Theorem 2.1. If $u_{0}$ belongs to $L^{2}(0, L)$ and $\eta<0$, then, problem (2.1) has a unique solution $u$ belonging to $C\left([0,+\infty) ; L^{2}(0, L)\right) \cap L_{\mathrm{loc}}^{2}\left(0,+\infty ; H_{0}^{2}(0, L)\right)$.

Proof. Using boundary conditions, we observe that the total energy associated to system

$$
E(t)=\frac{1}{2}\|u(t)\|^{2}
$$

satisfies the energy dissipation law

$$
\frac{\mathrm{d} E}{\mathrm{~d} t}=\frac{\eta}{2}\left|u_{x x}(0, t)\right|^{2}-\int_{0}^{L} a(x)|u(x, t)|^{2} \mathrm{~d} x \leq 0, \quad \forall t>0 .
$$

Consequently, it is sufficient to prove local (in time) existence and uniqueness. Global existence will then follow from $(2.2)$.

Let $A$ denote the closed linear operator $A v=-v^{\prime \prime \prime}-\eta v^{\prime \prime \prime \prime \prime \prime}$ defined on the dense domain $D(A) \subset L^{2}(0, L)$, where $D(A)=\left\{v \in H^{5}(0, L): v(0)=v(L)=v^{\prime}(0)=v^{\prime}(L)=v^{\prime \prime}(L)=0\right\}$.

On the other hand, we see that the adjoint operator $A^{*}$, is defined by $A^{*} w=w^{\prime \prime \prime}+\eta w^{\prime \prime \prime \prime \prime \prime}$, where $w$ belongs to $D\left(A^{*}\right)=\left\{w \in H^{5}(0, L): w(0)=w(L)=w^{\prime}(0)=w^{\prime}(L)=w^{\prime \prime}(0)=0\right\}$.

Using integration by parts we show that $A$ and $A^{*}$ are dissipative operators. Therefore, from classical results in semigroup theory (Pazy [17], Cor. 4.4, Chap. 1), we know that $A$ generates a strongly continuous semigroup of contractions in $L^{2}(0, L)$.

Let $\{S(t)\}_{t \geq 0}$ be the semigroup of contractions generated by $A$, then according to [25] (Thm. 1.1), we know that the semigroup of contractions $\{S(t)\}_{t \geq 0}$ satisfies the following properties:

$$
\begin{gathered}
\left\|S(t) u_{0}\right\| \leq\left\|u_{0}\right\|, \quad \forall t>0, \quad \forall u_{0} \in L^{2}(0, L) . \\
\left\|S(\cdot) u_{0}\right\|_{L^{2}\left(0, T ; H_{0}^{2}(0, L)\right)} \leq C\left\|u_{0}\right\|, \quad \forall T>0, \quad \forall u_{0} \in L^{2}(0, L) .
\end{gathered}
$$

Now, Theorem 2.1 follows from the following lemmas. 
Lemma 2.1. (1) If $u$ belongs to $L^{2}\left(0, T ; H_{0}^{2}(0, L)\right)$ then $u u_{x}$ belongs to $L^{1}\left(0, T ; H_{0}^{1}(0, L)\right)$ and the map $\Upsilon$ : $u \longrightarrow u u_{x}$ is continuous in the corresponding topologies.

(2) For $f \in L^{1}\left(0, T ; H_{0}^{1}(0, L)\right)$ the function defined by $v(\cdot, t)=\int_{0}^{t} S(t-s) f(\cdot, s) \mathrm{d} s$ belongs to $X_{T}=C\left([0, T] ; L^{2}(0, L)\right) \cap L^{2}\left(0, T ; H_{0}^{2}(0, L)\right)$ and the linear map $\Phi: f \longrightarrow v$ is continuous.

Proof. Since $u \in L^{2}\left(0, T ; H_{0}^{2}(0, L)\right)$, we have $u_{x} \in L^{2}\left(0, T ; H_{0}^{1}(0, L)\right)$ and hence, by classical results in one dimensional Sobolev spaces, $u u_{x}(\cdot, t)$ belongs to $H_{0}^{1}(0, L)$, for all $t \in(0, T)$ and moreover $\left(u u_{x}\right)_{x}=\left(u_{x}\right)^{2}+u u_{x x}$. So, using Sobolev embedding theorem, we have $H_{0}^{1}(0, L) \hookrightarrow L^{\infty}(0, L)$ and

$$
\int_{0}^{T}\left\|\left(u u_{x}\right)(\cdot, t)\right\|_{H_{0}^{1}(0, L)} \mathrm{d} t \leq C \int_{0}^{T}\|u(\cdot, t)\|_{H_{0}^{2}(0, L)}^{2} \mathrm{~d} t .
$$

To show the continuity of the map $\Upsilon$, we apply the triangular inequality and Hölder's inequality to obtain

$$
\|\Upsilon(u)-\Upsilon(w)\|_{L^{1}\left(0, T ; H_{0}^{1}(0, L)\right)} \leq C\left(\|u\|_{L^{2}\left(0, T ; H_{0}^{2}(0, L)\right)}+\|w\|_{L^{2}\left(0, T ; H_{0}^{2}(0, L)\right)}\right)\|u-w\|_{L^{2}\left(0, T ; H_{0}^{2}(0, L)\right)} .
$$

This shows that $\Upsilon$ is locally Lipschitz.

To prove item (2), we observe that $v$ is a mild solution of the following problem:

$$
\mid \begin{aligned}
& v_{t}+v_{x x x}+\eta v_{x x x x x}=f \\
& v(0, t)=v(L, t)=0 \\
& v_{x}(0, t)=v_{x}(L, t)=0 \\
& v_{x x}(L, t)=0 \\
& v(x, 0)=0
\end{aligned}
$$

Since, for $s \in(0, t)$,

$$
\|S(t-s) f(\cdot, s)\| \leq\|f(\cdot, s)\|
$$

it follows from Lebesgue's theorem that $v$ belongs to $C\left([0, T] ; L^{2}(0, L)\right)$ and moreover

$$
\|v(\cdot, t)\| \leq \int_{0}^{t}\|f(\cdot, s)\| \mathrm{d} s \leq\|f\|_{L^{1}\left(0, T ; H_{0}^{1}(0, L)\right)}, \quad \forall t \in[0, T] .
$$

On the other hand, integrating by parts in

$$
\int_{0}^{T} \int_{0}^{L} x v\left(v_{t}+v_{x x x}+\eta v_{x x x x x}\right) \mathrm{d} x \mathrm{~d} t=\int_{0}^{T} \int_{0}^{L} x v f \mathrm{~d} x \mathrm{~d} t
$$

we get

$$
\frac{1}{2} \int_{0}^{L} x|v(x, T)|^{2} \mathrm{~d} x+\frac{3}{2} \int_{0}^{T} \int_{0}^{L}\left|v_{x}(x, t)\right|^{2} \mathrm{~d} x \mathrm{~d} t-\eta \frac{5}{2} \int_{0}^{T} \int_{0}^{L}\left|v_{x x}(x, t)\right|^{2} \mathrm{~d} x \mathrm{~d} t=\int_{0}^{T} \int_{0}^{L} x(v f)(x, t) \mathrm{d} x \mathrm{~d} t .
$$

Hence, using (2.6),

$$
\begin{aligned}
\frac{3}{2} \int_{0}^{T} \int_{0}^{L}\left|v_{x}(x, t)\right|^{2} \mathrm{~d} x \mathrm{~d} t-\eta \frac{5}{2} \int_{0}^{T} \int_{0}^{L}\left|v_{x x}(x, t)\right|^{2} \mathrm{~d} x \mathrm{~d} t & \leq L\|f\|_{L^{1}\left(0, T ; H_{0}^{1}(0, L)\right)}\|v\|_{C\left([0, T] ; L^{2}(0, L)\right)} \\
& \leq L\|f\|_{L^{1}\left(0, T ; H_{0}^{1}(0, L)\right)}^{2}
\end{aligned}
$$

So, combining (2.6) and (2.7) we complete the proof of part (2) and the lemma holds as well. 
Lemma 2.2. The map $\Psi: X_{T} \longrightarrow X_{T}$ defined by

$$
\Psi(u)=S(t) u_{0}+\int_{0}^{t} S(t-s) u u_{x}(s) \mathrm{d} s
$$

is continuous. Moreover there exist $T>0$ and $R>0$ such that:

$\Psi$ is a contraction from $B_{R}$ to $B_{R}$, where $B_{R}$ is a ball of $X_{T}$.

Proof. The continuity of $\Psi$ follows by (2.3), (2.4) and Lemma 2.1 .

On the other hand, by Lemma 2.1 part (2) there exists $C_{1}>0$ such that:

$$
\|\Psi(u)-\Psi(v)\|_{X_{T}} \leq C_{1}\left\|u u_{x}-v v_{x}\right\|_{L^{1}\left(0, T ; H_{0}^{1}(0, L)\right)}
$$

So, applying triangular inequality and Hölder's inequality, we have

$$
\begin{aligned}
\|\Psi(u)-\Psi(v)\|_{X_{T}} \leq & \sqrt{2} C_{1}\|u-v\|_{L^{2}\left(0, T ; W^{1, \infty}(0, L)\right)}\|u\|_{L^{2}\left(0, T ; H_{0}^{2}(0, L)\right)} \\
& +\sqrt{2} C_{1}\|v\|_{L^{2}\left(0, T ; W^{1, \infty}(0, L)\right)}\|u-v\|_{L^{2}\left(0, T ; H_{0}^{2}(0, L)\right)}
\end{aligned}
$$

where, $W^{1, \infty}(0, L)=\left\{w \in L^{\infty}(0, L): w^{\prime} \in L^{\infty}(0, L)\right\}$.

Now, using Gagliardo-Niremberg inequality, it follows by (2.9) that there exists $C_{2}>0$ such that:

$$
\|\Psi(u)-\Psi(v)\|_{X_{T}} \leq C_{2} T^{\frac{1}{4}}\left\{\|u\|_{X_{T}}+\|v\|_{X_{T}}\right\}\|u-v\|_{X_{T}} .
$$

So, (2.10) shows that $\Psi$ is a contraction in the ball $B_{R}$ of $X_{T}$ if

$$
2 C_{2} T^{\frac{1}{4}} R<1
$$

We will complete the proof of lemma showing that, for a suitable choice of $R$ and $T$ satisfying the above inequality, the map $\Psi$ sends $B_{R}$ into itself.

Indeed, by (2.3), (2.4) and (2.10) we have

$$
\|\Psi(u)\|_{X_{T}} \leq C\left\|u_{0}\right\|+C_{2} T^{\frac{1}{4}}\|u\|_{X_{T}}^{2} .
$$

We consider $\bar{C}=\max \left\{C, C_{2}\right\}$ and we then take $R=2 \bar{C}\left\|u_{0}\right\|$. Therefore, in order to guarantee that the right side of (2.12) is less than $R$ and that (2.11) holds, we choose $T>0$ such that

$$
4 \bar{C}^{2} T^{\frac{1}{4}}|| u_{0} \| \leq 1
$$

So, we conclude the proof of Lemma 2.2

In order to prove Theorem 2.1, we observe that, using the variation of constants formula, system (2.1) may be written in the integral form (2.8).

Now, by Lemma 2.2 and Banach Fixed Point theorem we show the local existence and uniqueness of the solutions of (2.1), which completes the proof of the Theorem 2.1.

\section{EXPONENTIAL DECAY FOR KAWAHARA SYSTEM}

This section is devoted to analyze the exponential decay for $(\mathrm{K})$ system.

In Section 3.1, we study the case when the initial data is sufficiently small. We prove that, in this case, the system $(\mathrm{K})$ decays exponentially even in absence of damping $a$.

Section 3.2 is addressed to prove a general result of exponential decay for $(\mathrm{K})$ system and the gain of regularity of the solutions $u$. 


\subsection{Exponential decay of small amplitude solutions}

In this section we show the exponential decay of the energy associated with system (2.1), when the initial data $u_{0} \in L^{2}(0, L)$ is sufficiently small and the damping $a \equiv 0$.

Before proving the main result of this section, we need the following technical proposition:

Proposition 3.1. If $u$ is the solution of system (2.1) with initial data $u_{0}$ belonging to $L^{2}(0, L)$, then for $T>0$, there exists a positive constant $C_{2}=C_{2}(T, L, \eta)$ such that:

$$
\|u\|_{L^{2}\left(0, T ; H_{0}^{2}(0, L)\right)}^{2} \leq C_{2}\left[\left\|u_{0}\right\|^{2}+\left\|u_{0}\right\|^{4}\right]
$$

Proof. Multiplying the equation in $(2.1)$ by $x u$ and integrating in $(0, T) \times(0, L)$, we have

$$
\int_{0}^{T} \int_{0}^{L} u_{t} x u+u_{x x x} x u+\eta u_{x x x x x} x u+x u^{2} u_{x}+a(x) x u^{2} \mathrm{~d} x \mathrm{~d} t=0 .
$$

Integration by parts and boundary conditions of (2.1) give us

$$
\begin{aligned}
\frac{1}{2} \int_{0}^{L} x|u(x, T)|^{2} \mathrm{~d} x+\frac{3}{2} \int_{0}^{T} & \int_{0}^{L}\left|u_{x}(x, t)\right|^{2} \mathrm{~d} x \mathrm{~d} t-\eta \frac{5}{2} \int_{0}^{T} \int_{0}^{L}\left|u_{x x}(x, t)\right|^{2} \mathrm{~d} x \mathrm{~d} t \\
& +\int_{0}^{T} \int_{0}^{L} x a(x)|u(x, t)|^{2} \mathrm{~d} x \mathrm{~d} t \leq \frac{1}{2} \int_{0}^{L} x|u(x, 0)|^{2} \mathrm{~d} x+\frac{1}{3} \int_{0}^{T} \int_{0}^{L}|u(x, t)|^{3} \mathrm{~d} x \mathrm{~d} t .
\end{aligned}
$$

Now, by (2.2) and Sobolev embedding theorem we obtain:

$$
\frac{1}{3} \int_{0}^{T} \int_{0}^{L}|u(x, t)|^{3} \mathrm{~d} x \mathrm{~d} t \leq \frac{C}{3} \sqrt{T}\left\|u_{0}\right\|^{2}\|u\|_{L^{2}\left(0, T ; H_{0}^{2}(0, L)\right)} .
$$

So, replacing (3.3) in (3.2) and taking into account that $a(x) \geq 0$ and $\eta<0$, we obtain

$$
-\eta \frac{5}{2} \int_{0}^{T} \int_{0}^{L}\left|u_{x x}(x, t)\right|^{2} \mathrm{~d} x \mathrm{~d} t \leq \frac{L}{2}\left\|u_{0}\right\|^{2}+\frac{C}{3} \sqrt{T}\left\|u_{0}\right\|^{2}\|u\|_{L^{2}\left(0, T ; H_{0}^{2}(0, L)\right)}
$$

Therefore,

$$
\|u\|_{L^{2}\left(0, T ; H_{0}^{2}(0, L)\right)}^{2} \leq \frac{4 L}{-10 \eta}\left\|u_{0}\right\|^{2}+\frac{4 C^{2} T}{225 \eta^{2}}\left\|u_{0}\right\|^{4}
$$

which concludes the proof. 
Now, we are ready to prove the main theorem of this subsection.

Theorem 3.1 (stabilization of small amplitude solutions). Let $a \equiv 0$ in the problem (2.1). Then, there exists $\delta>0$ such that if the initial data $u_{0} \in L^{2}(0, L)$ of the problem (2.1) satisfies $\left\|u_{0}\right\| \leq \delta$, the energy $E(t)=\frac{1}{2}\|u(t)\|^{2}$, associated to the problem, decays exponentially for all lengths $L$.

More precisely, there exist $c>0$ and $\mu>0$ such that: $E(t) \leq c \mathrm{e}^{-\mu t} E(0)$.

Proof. According to [25] (Thm. 2.2) and [26] (remarks) the linear semigroup $\{S(t)\}_{t \geq 0}$ satisfies

$$
\|S(t)\| \leq c \mathrm{e}^{-\mu t}
$$

even when $a \equiv 0$.

Let $T>0$ be such that

$$
\rho=\|S(T)\|_{\mathcal{L}\left(L^{2}(0, L), L^{2}(0, L)\right)}<1 .
$$

The solution of problem (2.1) may be written in the integral form defined by (2.8). In particular, for $t=T$ we have

$$
u(T)=S(T) u_{0}+\int_{0}^{T} S(T-s) u u_{x}(s) \mathrm{d} s .
$$

Therefore, by (2.5) and (3.4), we obtain

$$
\|u(T)\| \leq \rho\left\|u_{0}\right\|+C\|u\|_{L^{2}\left(0, T ; H_{0}^{2}(0, L)\right)}^{2} .
$$

Replacing the inequality (3.1) in (3.5) it follows that

$$
\|u(T)\| \leq\left\|u_{0}\right\|\left\{\rho+C_{3}\left[\left\|u_{0}\right\|+\left\|u_{0}\right\|^{3}\right]\right\}
$$

where $C_{3}=C C_{2}$.

Now, taking

$$
C_{3}\left[\left\|u_{0}\right\|+\left\|u_{0}\right\|^{3}\right]<\frac{1-\rho}{2}
$$

we see that

$$
\|u(T)\| \leq \frac{1+\rho}{2}\left\|u_{0}\right\|
$$

The above inequality together with semigroup property implies the exponential decays of the energy when the initial data satisfies (3.6).

Remark 3.1. The argument used to prove the Theorem 3.1 provides an exponential decay of the energy only when the size of the initial data is small enough. This argument fails for large solutions, whose analysis will be performed in the next subsection.

\subsection{The exponential decay for large solutions}

The aim of this subsection is to examine the locally uniform exponential decay of the energy associated to the problem (2.1) for large solutions. We employ the method developed in [25], however, we may not apply Holmgren's principle since we have now a semi-linear problem. Our analysis follows the same method used in [15]. In order to conclude we need an important result, named unique continuation principle, which we state below. 


\section{Unique Continuation Principle (UCP) for Kawahara system}

Let $\omega$ be an open, non-empty subset of $(0, L)$.

If $v \in L^{2}\left(0, T ; H^{5}(0, L)\right) \cap L^{2}\left(0, T ; H_{0}^{2}(0, L)\right) \cap L^{\infty}\left(0, T ; L^{2}(0, L)\right)$ solves

$$
\mid \begin{aligned}
& v_{t}+v_{x x x}+\eta v_{x x x x x}+\lambda v v_{x}=0 \quad \text { in }(0, L) \times(0, T) \\
& v(0, t)=v(L, t)=0 \quad \text { for all } t \in(0, T) \\
& v_{x}(0, t)=v_{x}(L, t)=0 \quad \text { for all } t \in(0, T) \\
& v_{x x}(L, t)=0 \quad \text { for all } t \in(0, T) \\
& v \equiv 0 \quad \text { in } \omega \times(0, T)
\end{aligned}
$$

with $\lambda>0$ and $T>0$, then necessarily $v \equiv 0$ in $(0, L) \times(0, T)$.

The proof of the UCP follows directly from the following result due to Saut and Scheurer [22] (Thm. 4.2).

Let $\mathcal{O}$ be the cylinder $\Omega \times(-T, T), \Omega$ open interval in $\mathbb{R}$. Let $L$ be defined by

$$
L=i D_{t}+\alpha i^{2 k+1} D^{2 k+1}+R(x, t, D), \quad \alpha \neq 0, \quad D=\frac{1}{i} \frac{\partial}{\partial x}
$$

with $R(x, t, D)=\sum_{j=0}^{2 k} r_{j}(x, t) D^{j}$. We assume that $r_{j} \in L^{\infty}\left(-T, T ; L_{\mathrm{loc}}^{2}(\Omega)\right)$.

Let $u \in L^{2}\left(-T, T ; H_{\mathrm{loc}}^{2 k+1}(\Omega)\right)$ be a solution of $L u=0$, which vanishes in some open set $\mathcal{O}_{1} \subset \mathcal{O}$. Then $u$ vanishes in the horizontal component of $\mathcal{O}_{1}$.

In our case, we take $k=2$ and by means of a change of variables, we consider $R(x, t, D)=i D^{3}+i r(x, t) D$ with $r(x, t)=\widetilde{\lambda} \widetilde{u}$, where $\widetilde{u} \in L^{2}\left(-T, T ; H_{\text {loc }}^{5}(\Omega)\right)$ is the solution of (3.7) in the new variables.

Now, since UCP holds, we have the following result.

Theorem 3.2 (stabilization). Assume the open subset $\omega$ of $(0, L)$ is such that the (UCP) above holds. Let $a=a(x)$ be a non-negative function belonging to $L^{\infty}(0, L)$ such that $a(x) \geq a_{0}>0$ a.e. in $\omega$. Then, for all $R>0$, there exist $c(R)=c>0$ and $\mu(R)=\mu>0$ such that

$$
E(t) \leq c\left\|u_{0}\right\|^{2} \mathrm{e}^{-\mu t}
$$

for all $t \geq 0$ and any solution of $(2.1)$ with $u_{0} \in L^{2}(0, L)$ and $\left\|u_{0}\right\| \leq R$.

Proof. Multiplying the equation in $(2.1)$ by $(T-t) u$ and integrating on $(0, L) \times(0, T)$ we have

$$
\frac{1}{2}\left\|u_{0}\right\|^{2} \leq \frac{1}{2 T} \int_{0}^{T} \int_{0}^{L}|u(x, t)|^{2} \mathrm{~d} x \mathrm{~d} t-\frac{\eta}{2} \int_{0}^{T}\left|u_{x x}(0, t)\right|^{2} \mathrm{~d} t+\int_{0}^{T} \int_{0}^{L} a(x)|u(x, t)|^{2} \mathrm{~d} x \mathrm{~d} t
$$

since $\int_{0}^{T} \int_{0}^{L}(T-t) u^{2} u_{x} \mathrm{~d} x \mathrm{~d} t=0$.

So, by semigroup property, it is sufficient to prove that for any $T>0$ and $R>0$, there exists a positive constant $c_{1}(R, T)=c_{1}$ such that

$$
\frac{1}{2} \int_{0}^{T} \int_{0}^{L}|u(x, t)|^{2} \mathrm{~d} x \mathrm{~d} t \leq c_{1}\left\{\frac{-\eta}{2} \int_{0}^{T}\left|u_{x x}(0, t)\right|^{2} \mathrm{~d} t+\int_{0}^{T} \int_{0}^{L} a(x)|u(x, t)|^{2} \mathrm{~d} x \mathrm{~d} t\right\}
$$

for any solution $u$ of $(2.1)$ with $\left\|u_{0}\right\| \leq R$. Again we argue by contradiction. Suppose that (3.10) does not hold. 
Then there exists a sequence $\left\{u_{n}\right\}$ of functions in $L^{\infty}\left(0, T ; L^{2}(0, L)\right) \cap L^{2}\left(0, T ; H_{0}^{2}(0, L)\right)$ solutions of (2.1), satisfying $\left\|u_{n}(\cdot, 0)\right\| \leq R$ and such that

$$
\lim _{n \rightarrow \infty} \frac{\int_{0}^{T} \int_{0}^{L}\left|u_{n}(x, t)\right|^{2} \mathrm{~d} x \mathrm{~d} t}{\left[-\eta \int_{0}^{T}\left|\left(u_{n}\right)_{x x}(0, t)\right|^{2} \mathrm{~d} t+2 \int_{0}^{T} \int_{0}^{L} a(x)\left|u_{n}(x, t)\right|^{2} \mathrm{~d} x \mathrm{~d} t\right]}=+\infty .
$$

Let $\lambda_{n}=\sqrt{\int_{0}^{T} \int_{0}^{L}\left|u_{n}(x, t)\right|^{2} \mathrm{~d} x \mathrm{~d} t}$ and $v_{n}(x, t)=\frac{u_{n}(x, t)}{\lambda_{n}}$. Clearly, for each $n \in \mathbb{N}$, the functions $v_{n}$ satisfy:

$$
\mid \begin{array}{ll}
\left(v_{n}\right)_{t}+\left(v_{n}\right)_{x x x}+\eta\left(v_{n}\right)_{x x x x x}+\lambda_{n}\left(v_{n}\right)\left(v_{n}\right)_{x}+a(x) v_{n}=0 & \text { in }(0, L) \times(0, T) \\
v_{n}(0, t)=v_{n}(L, t)=0 \quad \text { for all } t \in(0, T) & \\
\left(v_{n}\right)_{x}(0, t)=\left(v_{n}\right)_{x}(L, t)=0 \quad \text { for all } t \in(0, T) & \text { for all } t \in(0, T)
\end{array}
$$

$$
\int_{0}^{T} \int_{0}^{L}\left|v_{n}(x, t)\right|^{2} \mathrm{~d} x \mathrm{~d} t=1
$$

and

$$
\lim _{n \rightarrow \infty}\left\{-\eta \int_{0}^{T}\left|\left(v_{n}\right)_{x x}(0, t)\right|^{2} \mathrm{~d} t+2 \int_{0}^{T} \int_{0}^{L} a(x)\left|v_{n}(x, t)\right|^{2} \mathrm{~d} x \mathrm{~d} t\right\}=0 .
$$

Using (3.9), (3.13) and (3.14) we can prove that $v_{n}(x, 0)$ is a bounded sequence in $L^{2}(0, L)$. Then, by (3.1), there exists $M(L, \eta, T)=M>0$ such that:

$$
\left\|v_{n}\right\|_{L^{2}\left(0, T ; H_{0}^{2}(0, L)\right)} \leq M .
$$

Now, since $\left\{v_{n}\right\}$ belongs to $L^{\infty}\left(0, T ; L^{2}(0, L)\right) \cap L^{2}\left(0, T ; H_{0}^{2}(0, L)\right)$ and $v_{n}(x, 0)$ is a bounded sequence in $L^{2}(0, L)$, it follows from (3.15) and Sobolev embedding property that

$$
\left\|v_{n}\left(v_{n}\right)_{x}\right\|_{L^{2}((0, L) \times(0, T))} \leq c_{1}\left\|v_{n}\right\|_{L^{\infty}\left(0, T ; L^{2}(0, L)\right)}\left\|v_{n}\right\|_{L^{2}\left(0, T ; H_{0}^{2}(0, L)\right)} \leq C
$$

for some constant $C(L, \eta, T)=C>0$.

So, as $\left\{\lambda_{n}\right\}$ is a bounded sequence (because, by assumption, $\left\|u_{n}(x, 0)\right\| \leq R$ ), we conclude, by (3.12), (3.13), (3.15) and (3.16), that $\left\{\left(v_{n}\right)_{t}\right\}$ is bounded in $L^{2}\left(0, T ; H^{-3}(0, L)\right)$.

Since the embedding $H_{0}^{2}(0, L) \hookrightarrow L^{2}(0, L)$ is compact, it follows by using classical compactness results that $v_{n}$ is relatively compact in $L^{2}((0, L) \times(0, T))$. By extracting subsequences we obtain

$$
v_{n} \rightarrow v \text { strongly in } L^{2}((0, L) \times(0, T)) .
$$

Here, to simplify the notation, we denote the subsequence by the same index $n$.

By (3.13), we have

$$
\|v\|_{L^{2}((0, L) \times(0, T))}=1 .
$$

Since $a \in L^{\infty}(0, L)$, then by (3.14) and (3.17) we deduce

$$
\begin{aligned}
0 & =\liminf _{n \rightarrow \infty}\left\{-\eta \int_{0}^{T}\left|\left(v_{n}\right)_{x x}(0, t)\right|^{2} \mathrm{~d} t+2 \int_{0}^{T} \int_{0}^{L} a(x)\left|v_{n}(x, t)\right|^{2} \mathrm{~d} x \mathrm{~d} t\right\} \\
& \geq\left\{-\eta \int_{0}^{T}\left|v_{x x}(0, t)\right|^{2} \mathrm{~d} t+2 \int_{0}^{T} \int_{0}^{L} a(x)|v(x, t)|^{2} \mathrm{~d} x \mathrm{~d} t\right\}
\end{aligned}
$$


which guarantees that $a(x) v \equiv 0$ and so $v \equiv 0$ in $\omega \times(0, T)$, furthermore, $v_{x x}(0, t)=0$ in $(0, T)$. More precisely, the limit $v \in L^{2}\left(0, T ; H_{0}^{2}(0, L)\right) \cap L^{\infty}\left(0, T ; L^{2}(0, L)\right)$ and satisfies

$$
\mid \begin{array}{lc}
v_{t}+v_{x x x}+\eta v_{x x x x x}+\lambda v v_{x}=0 & \text { in }(0, L) \times(0, T) \\
v(0, t)=v(L, t)=0 & \text { for all } t \in(0, T) \\
v_{x}(0, t)=v_{x}(L, t)=0 & \text { for all } t \in(0, T) \\
v_{x x}(0, t)=v_{x x}(L, t)=0 & \text { for all } t \in(0, T) \\
v(x, t)=0 \quad(x, t) \in \omega \times(0, T)
\end{array}
$$

where $\lambda=\lim _{n \rightarrow \infty} \lambda_{n}$ (we consider a subsequence also denoted by $\lambda_{n}$ ).

We distinguish two situations:

(a) $\lambda=0$

In this case, the limit $v$ satisfies a linear problem as in [25], then by Holmgren's Uniqueness Theorem, we obtain $v \equiv 0$ in $(0, T) \times(0, L)$. This contradicts (3.18) and, consequently, (3.10) holds.

(b) $\lambda>0$

In this case, the limit $v$ solves (3.19) and to prove (3.10) we need the following gain of regularity result:

Proposition 3.2. (gain of regularity for (3.19)). If $v \in L^{2}\left(0, T ; H_{0}^{2}(0, L)\right) \cap L^{\infty}\left(0, T ; L^{2}(0, L)\right)$ and satisfies problem (3.19), then $v$ belongs to $L^{2}\left(0, T ; H^{5}(0, L)\right)$.

We shall prove this proposition in next section.

Then, assuming the $(\mathrm{UCP})$ holding for the subset $\omega$, we have $v \equiv 0$ in $(0, T) \times(0, L)$ and this is a contradiction.

Remark 3.2. We observe that we have used in an essential way the assumption that the initial data are in a ball of radius $R$. Otherwise, in the contradiction argument, we would have to consider the case $\lambda_{n} \rightarrow+\infty$. This has only been done by Zuazua [28] in the context of semi-linear wave equation with localized damping under suitable structural assumptions on the nonlinearity.

Remark 3.3. It is important to analyze the proof of Theorem 3.2 to understand the amount of damping necessary to guarantee the exponential decay of the energy.

Going back to the proof of Theorem 3.2, we observe that the limit solution $v$ satisfies the following conditions:

$$
\begin{aligned}
v_{x x}(0, t) & =0 & & \text { for all } t>0 \\
v & \equiv 0 & & \text { in } \omega \times(0, T) .
\end{aligned}
$$

Then using, Proposition 3.2 and the UCP we obtain the contradiction.

So, we can observe that to obtain the above conditions we only need a very weak amount of additional damping to stabilize the Kawahara system. In fact, we do not need a damping mechanism that dissipates the $L^{2}$-norm as $a(\cdot) u$ does, it is suffice to dissipate some $H^{-s}$-norm to guarantee the exponential decay of the energy associated to Kawahara system.

It is important to note that Theorem 3.2 proves the exponential decay of the energy, associated to nonlinear (K) system, under two important assumptions, the gain of regularity for (3.19) and the UCP. In the next section we shall prove the gain of regularity.

\section{Gain of Regularity for the problem (3.19)}

In this section we prove the Proposition 3.2. The arguments used here are similar to [16]. So, we only give a sketch of the proof. 
Proof. We consider the following system:

$$
\mid \begin{aligned}
& w_{t}+w_{x x x}+\eta w_{x x x x x}+\lambda(v w)_{x}=0 \quad \text { in }(0, L) \times(0, T) \\
& w(0, t)=w(L, t)=0 \quad \text { for all } t \in(0, T) \\
& w_{x}(0, t)=w_{x}(L, t)=0 \quad \text { for all } t \in(0, T) \\
& w_{x x}(L, t)=0 \quad \text { for all } t \in(0, T) \\
& w(x, 0)=w_{0}
\end{aligned}
$$

where $v$ belongs to $L^{2}\left(0, T ; H_{0}^{2}(0, L)\right) \cap L^{\infty}\left(0, T ; L^{2}(0, L)\right)$ and is the solution of system $(2.1)$ with initial data $v_{0}$ belonging to $L^{2}(0, L)$ and $\lambda>0$.

Lemma 4.1. If $w_{0}$ belongs to $L^{2}(0, L)$ and $v$ satisfies the above assumptions, then system (4.1) has a unique solution $w$ belonging to $L^{2}\left(0, T ; H_{0}^{2}(0, L)\right) \cap L^{\infty}\left(0, T ; L^{2}(0, L)\right)$.

Proof. As it was done in Theorem 2.1, let $\{S(t)\}_{t \geq 0}$ be the semigroup of contractions generated by the operator $A$.

Using the variation of constants formula, system (4.1) may be written by:

$$
\Psi(w)=S(t) w_{0}+\int_{0}^{t} S(t-s)(w v)_{x} \mathrm{~d} s .
$$

In the same way that in Theorem 2.1, we may prove that the above map is a contraction in a ball of $X_{T}$ and then we use the Banach fixed point theorem.

Now, using multipliers techniques we prove that this solution exists globally.

Lemma 4.2. As in Lemma 4.1, if $w$ solves system (4.1) with $w_{0}$ in $L^{2}(0, L)$, then there exists a positive constant $C=C\left(T,\left\|v_{0}\right\|_{L^{2}(0, L)}\right)$ such that:

$$
\left\|w_{0}\right\|_{L^{2}(0, L)}^{2} \leq C\left(\int_{0}^{T}\left|w_{x x}(0, t)\right|^{2} \mathrm{~d} t+\left\|w_{0}\right\|_{H^{-5}(0, L)}^{2}\right)
$$

Proof. To prove the inequality (4.3) we combine multiplier techniques and the compactness-uniqueness argument in similar way as we have used in Theorem 3.2.

Now, we are in conditions to prove Proposition 3.2 .

Differentiating equation in (3.19) with respect to $t$ and taking $w=v_{t}$, we obtain the following system:

$$
\mid \begin{aligned}
& w_{t}+w_{x x x}+\eta w_{x x x x x}+\lambda(v w)_{x}=0 \quad \text { in }(0, L) \times(0, T) \\
& w(0, t)=w(L, t)=0 \quad \text { for all } t \in(0, T) \\
& w_{x}(0, t)=w_{x}(L, t)=0 \quad \text { for all } t \in(0, T) \\
& w_{x x}(L, t)=0 \quad \text { for all } t \in(0, T)
\end{aligned}
$$

with $w_{0}(x)=w(x, 0)=v_{t}(x, 0)=-v_{0, x x x}+\eta v_{0, x x x x x}+\lambda v_{0} v_{0, x} \in H^{-5}(0, L)$.

Moreover, we have $w_{x x}(0, t)=0$ and $w(x, t)=0,(x, t) \in \omega \times(0, T)$. 
Now, according to Lemma 4.2 we obtain $w_{0} \in L^{2}(0, L)$. Then, combining Lemma 4.1 and system (3.19) we get

and by (3.19) we have:

$$
v_{t}=w \in L^{\infty}\left(0, T ; L^{2}(0, L)\right) \cap L^{2}\left(0, T ; H_{0}^{2}(0, L)\right)
$$

$$
\eta v_{x x x x x}=-v_{t}-v_{x x x}-\lambda v v_{x} \quad \text { in }(0, L) \times(0, T) .
$$

So, by (4.5) and (4.6), $v_{x x x x x}$ belongs to $L^{2}\left(0, T ; H^{-1}(0, L)\right)$ and therefore $v_{x x x x}$ is in $L^{2}\left(0, T ; L^{2}(0, L)\right)$, which implies $v_{x x x}$ in $L^{2}\left(0, T ; L^{2}(0, L)\right)$. Again, using $(4.6)$ we can conclude that $v_{x x x x x}$ belongs to $L^{2}\left(0, T ; L^{2}(0, L)\right)$, which proves Proposition 3.2 .

\section{FinAl REMARKS}

As pointed out in the introduction we present now a brief discussion about the proof of the presented results if we add the extra term $u_{x}$ to the Kawahara system $(\mathrm{K})$.

(1) In the existence and uniqueness (Thm. 2.1) the same arguments apply to the new system without any modification.

(2) In the exponential decay for small amplitude solutions data (Prop. 3.1), there is a slightly difference in the constant $C_{2}$ of inequality (3.1). In fact, due to $u_{x}$ term, we have to deal with $\|u\|_{L^{2}\left(0, T ; L^{2}(0, L)\right)}$ which is also bounded by $\left\|u_{0}\right\|$.

(3) In the Unique Continuation Principle for Kawahara system, we only need to take $R(x, t, D)=i D^{3}+$ $i D+i r(x, t) D$ instead of $R(x, t, D)=i D^{3}+i r(x, t) D$.

(4) In the exponential decay for large solutions (Thm. 3.2)) and in the gain of regularity for problem (3.19) (Lems. 4.1 and 4.2), the same arguments apply to the new system without any modification.

The problem of decay of solutions for Kawahara system (2.1) makes sense even in absence of the damping $a$, that is, when $a \equiv 0$. In fact, according with the energy dissipation law we have:

The total energy associated with system (2.1) is defined by

$$
E(t)=\frac{1}{2} \int_{0}^{L}|u(x, t)|^{2} \mathrm{~d} x=\frac{1}{2}\|u(t)\|^{2} .
$$

Using the boundary conditions in (2.1) we obtain that

$$
\frac{\mathrm{d} E}{\mathrm{~d} t}=\frac{\eta}{2}\left|u_{x x}(0, t)\right|^{2} \leq 0, \forall t>0
$$

Therefore, in this case, $E(t)$ is a nonincreasing function.

Now, it is natural to formulate the following questions:

- Does the energy $E(t) \rightarrow 0$ as $t \rightarrow+\infty$ ?

- Is it possible to find a rate of decay of the energy?

In this case, that is when $a \equiv 0$, it is important to observe that Theorem 3.2 holds if the following unique continuation principle can be proved:

If $v \in L^{2}\left(0, T ; H^{5}(0, L)\right) \cap L^{2}\left(0, T ; H_{0}^{2}(0, L)\right) \cap L^{\infty}\left(0, T ; L^{2}(0, L)\right)$ solves

$$
\mid \begin{array}{lc}
v_{t}+v_{x x x}+\eta v_{x x x x x}+\lambda v v_{x}=0 & \text { in }(0, L) \times(0, T) \\
v(0, t)=v(L, t)=0 & \text { for all } t \in(0, T) \\
v_{x}(0, t)=v_{x}(L, t)=0 & \text { for all } t \in(0, T) \\
v_{x x}(0, t)=v_{x x}(L, t)=0 & \text { for all } t \in(0, T)
\end{array}
$$

with $\lambda>0$ and $T>0$, then necessarily $v \equiv 0$ in $(0, L) \times(0, T)$. 
As far as we know, the above unique continuation principle remains to be done.

Coron and Crépeau [6] studied the boundary controllability of the nonlinear KdV equation with Dirichlet boundary condition on an interval with critical length and they proved that the nonlinear term gives the local controllability around the origin.

On the other hand, we can consider the Kawahara system with more general nonlinearity, replacing the non linear term $u u_{x}$, in the equation, by $b(u) u_{x}$, where the real function $b$ is a smooth function satisfying some growth condition, as it was studied for the KdV system by Rosier and Zhang in [20]. There, the authors proved existence, uniqueness and global stabilization for the Generalized KdV equation. Linares and Pazoto [12] analyzed the existence and the locally uniform exponential decay of solutions of the critical generalized Korteweg-de Vries equation in a bounded interval with a localized damping term. There the nonlinear term it is given by $u^{4} u_{x}$ and the initial data is small.

The study of the Kawahara system with more general nonlinearity, that is the existence, uniqueness and stabilization of solutions is an open problem.

In the Kawahara system, as indicated in Remark 3.3, the damping term $a(\cdot) u$ may be replaced by any other weaker damping mechanism, for instance, $B u$, where $B$ is a bounded operator in $L^{2}(0, L)$ such that $B u \equiv 0$ implies that $u \equiv 0$ in $\omega$.

In Massarolo et al. [14] has been obtained, for KdV system, the locally uniform energy decay at an exponential rate with a very weak localized dissipation namely the $H^{-1}$ norm. Indeed, they considered the damping given by the bounded linear operator $B=1_{\omega}(-\triangle)^{-1}$, where $\omega$ is a non-empty open subset of $(0, L)$.

We believe that similar problem can be solved for Kawahara system without majors difficulties.

Acknowledgements. The authors thank E. Zuazua for his suggestions and comments.

\section{REFERENCES}

[1] T.B. Benjamin, J.L. Bona and J.J. Mahony, Model equations for long waves in nonlinear dispersive systems. Phil. Trans. R. Soc. A $272(1972) 47-78$.

[2] N.G. Berloff and L.N. Howard, Solitary and periodic solutions for nonlinear nonintegrable equations. Stud. Appl. Math. 99 (1997) 1-24.

[3] H.A. Biagioni and F. Linares, On the Benney-Lin and Kawahara equations. J. Math. Anal. Appl. 211 (1997) 131-152.

[4] J.L. Bona and H. Chen, Comparison of model equations for small-amplitude long waves. Nonlinear Anal. 38 (1999) 625-647.

[5] T.J. Bridges and G. Derks, Linear instability of solitary wave solutions of the Kawahara equation and its generalizations. SIAM J. Math. Anal. 33 (2002) 1356-1378.

[6] J.M. Coron and E. Crépeau, Exact boundary controllability of a nonlinear KdV equation with critical lenghts. J. Eur. Math. Soc. 6 (2004) 367-398.

[7] G.G. Doronin and N.A. Larkin, Kawahara equation in a bounded domain. Discrete Continuous Dyn. Syst., Ser. B 10 (2008) 783-799.

[8] H. Hasimoto, Water waves. Kagaku 40 (1970) 401-408 [in Japanese].

[9] T. Kakutani and H. Ono, Weak non-linear hydromagnetic waves in a cold collision-free plasma. J. Phys. Soc. Japan 26 (1969) $1305-1318$.

[10] T. Kawahara, Oscillatory solitary waves in dispersive media. J. Phys. Soc. Japan 33 (1972) 260-264.

[11] F. Linares and J.H. Ortega, On the controllability and stabilization of the linearized Benjamin-Ono equation. ESAIM: COCV 11 (2005) 204-218.

[12] F. Linares and A.F. Pazoto, On the exponential decay of the critical generalized Korteweg-de Vries with localized damping. Proc. Amer. Math. Soc. 135 (2007) 1515-1522.

[13] J.L. Lions, Contrôlabilité exacte, perturbations et stabilisation de systèmes distribués, Tome 1: Contrôlabilité Exacte, in RMA 8, Masson, Paris, France (1988).

[14] C.P. Massarolo, G.P. Menzala and A.F. Pazoto, On the uniform decay for the Korteweg-de Vries equation with weak damping. Math. Meth. Appl. Sci. 30 (2007) 1419-1435.

[15] G.P. Menzala, C.F. Vasconcellos and E. Zuazua, Stabilization of the Korteweg-de Vries equation with localized damping. Quarterly Applied Math. LX (2002) 111-129.

[16] A.F. Pazoto, Unique continuation and decay for the Korteweg-de Vries equation with localized damping. ESAIM: COCV 11 (2005) 473-486. 
[17] A. Pazy, Semigroups of linear operators and applications to partial differential equations. Springer-Verlag, New York, USA (1983).

[18] J. Rauch and M. Taylor, Exponential decay of solutions to hyperbolic equations in bounded domains. Indiana Univ. Math. J. 24 (1974) 79-86.

[19] L. Rosier, Exact boundary controllability for the Korteweg-de Vries equation on a bounded domain. ESAIM: COCV 2 (1997) $33-55$.

[20] L. Rosier and B.Y. Zhang, Global stabilization of the generalized Korteweg-de Vries equation posed on a finite domain. SIAM J. Contr. Opt. 45 (2006) 927-956.

[21] D.L. Russell and B.Y. Zhang, Exact controllability and stabilization of the Korteweg-de Vries equation. Trans. Amer. Math. Soc. 348 (1996) 1515-1522.

[22] J.C. Saut and B. Scheurer, Unique continuation for some evolution equations. J. Diff. Equation 66 (1987) 118-139.

[23] G. Schneider and C.E. Wayne, The rigorous approximation of long-wavelength capillary-gravity waves. Arch. Ration. Mech. Anal. 162 (2002) 247-285.

[24] J. Topper and T. Kawahara, Approximate equations for long nonlinear waves on a viscous fluid. J. Phys. Soc. Japan 44 (1978) 663-666.

[25] C.F. Vasconcellos and P.N. da Silva, Stabilization of the linear Kawahara equation with localized damping. Asymptotic Anal. 58 (2008) 229-252.

[26] C.F. Vasconcellos and P.N. da Silva, Erratum of the Stabilization of the linear Kawahara equation with localized damping. Asymptotic Anal. (to appear).

[27] E. Zuazua, Contrôlabilité Exacte de Quelques Modèles de Plaques en un Temps Arbitrairement Petit. Appendix in [13], $165-191$.

[28] E. Zuazua, Exponential decay for the semilinear wave equation with locally distribued damping. Comm. Partial Diff. Eq. 15 (1990) 205-235. 